www.jmscr.igmpublication.org

Impact Factor (SJIF): 6.379

Index Copernicus Value: 79.54

ISSN (e)-2347-176x ISSN (p) 2455-0450

crossrefDOI: https://dx.doi.org/10.18535/jmscr/v6i10.88

Journal Of Medical Science And Clinical Research

IGM Publication

An Official Publication of IGM Publication

\title{
A Comparative Prospective Study of Platelet Rich Plasma vs Corticosteroid Injection in Lateral Epicondylitis
}

\author{
Authors \\ Dr Anwar. A ${ }^{* 1}$, Dr Vikram Shetty ${ }^{2}$, Dr Siddarth M. Shetty ${ }^{3}$, Dr Y. Nishanth Shetty ${ }^{4}$ \\ ${ }^{* 1}$ Post Graduate in Orthopaedics K.S.Hegde Medical Academy, NITTE University \\ ${ }^{2}$ Professor Department of Orthopaedics K.S.Hegde Medical Academy, NITTE University \\ ${ }^{3}$ Additional Professor, Department of Orthopaedics, K.S.Hegde Medical Academy, NITTE University \\ ${ }^{4}$ Senior Registrar Department of Orthopaedics, VPS Lakeshore Hospital, Kochi
}

\begin{abstract}
Introduction: Lateral epicondylitis, is seen to affect $1 \%$ to $3 \%$ of the general population in the $3^{\text {rd }}$ and $4^{\text {th }}$ decade of life and is one of the most common causes for elbow pain. Various treatment modalities have been used in its treatment including analgesics and immobilisation, wrist bracing, elbow bracing, local corticosteroid injection, shockwave therapy and modifying poor technique in sport or work and newer treatments like injecting Platelet Rich Plasma.

Aim: To study \& compare the functional and subjective outcomes in patients with lateral epicondylitis treated with corticosteroid injections and platelet Rich plasma

Materials \& Methods: 40 patients were divided equally into 2 groups based on block randomization, with one group receiving PRP and the other Corticosteroid injection. All the patients were assessed using the $V A S$ and the LES score which was taken pre injection and post injection at 6 weeks, 3 months and 6 months respectively.

Results: At the end of the follow up, patients in both the groups were assessed using the VAS and the LES scoring system and statistically significant values were obtained $(P<0.001)$. The results obtained showed that the patients who received PRP injections had better clinical outcome than those who received the corticosteroid injection.

Conclusion: Both PRP and Corticosteroids were effective in the treatment of lateral epicondylitis but PRP proved to be more effective modality in the treatment of lateral epicondylitis with a statistically significant better functional outcome and better pain relief.

Keywords: Tennis elbow, PRP, Platelet Rich Plasma.
\end{abstract}

\section{Introduction}

Lateral epicondylitis, commonly referred to as 'tennis elbow' is seen to affect $1 \%$ to $3 \%$ of the general population in the $3^{\text {rd }}$ and $4^{\text {th }}$ decade of life. It is one of the most common causes for elbow pain.
The pathogenesis of an overuse injury is thought to be a result from cumulative micro trauma that weakens the structural and vascular elements of the tendon ${ }^{1}$. Micro trauma to a mechanical structure occurs even if the loads are within the material's strength limits, and is due to fatigue after repetitive loads. If the muscle is weak or 
fatigued, the energy absorbing capacity of the whole muscle-tendon unit is reduced, and tendon stresses will increase ${ }^{2}$.

In chronic Lateral epicondylitis (pain duration more than 3 months) there occurs vasodilatation and plasma extravasations, but without presence of any inflammatory cells.

Treatment modalities for the treatment of lateral epicondylitis include analgesics and immobilisation. $90 \%$ of cases resolve spontaneously within 6-12 months. Other modalities include wrist bracing, elbow bracing, local corticosteroid injection, shockwave therapy and modifying poor technique in sport or work ${ }^{3}$ injection treatment have been used in the treatment for Lateral Epicondylitis. Corticosteroid Injection has been used in the treatment of Lateral Epicondylitis ${ }^{1,4}$. But the treatment with steroid is only seen effective in the early management. It has also got adverse side effects like atrophy and permanent structural changes of the tendon ${ }^{1,4}$.

Another alternative method is Platelet Rich Plasma (PRP) injection by providing safe and natural healing. Platelets release many bioactive proteins responsible for attracting macrophages, mesenchymal stem cells and osteoblasts which helps in tissue regeneration and wound healing ${ }^{1,2}$. Platelet rich plasma (PRP) is defined as a volume of the plasma fraction of autologous blood having a platelet concentration above base line. Platelet increase should be minimum increase of 4 times the baseline $e^{1,5}$.

\section{Materials and Method}

Prospective comparative study performed at the orthopedics department in K.S. Hegde Hospital, Mangalore from August 2015 to November 2017 are included in the study after explaining the procedure with consent by block randomization of 40 patients with 20 in each group.

\section{Inclusion criteria}

- Patients in the age group of 20-70 years

- Patients with symptoms of lateral epicondylitis for more than 3 months not responding to 6 weeks of conservative management

\section{Exclusion criteria}

- Diabetic patients

- Patients suffering with rheumatoid arthritis

- Patients with history of trauma or surgery to the lateral epicondyle

- Patients who have previously received an injection at the lateral epicondyle in the last 3 months

- Patients suspicion of nerve involvement.

\section{Technique, data Collection and Analysis}

- Corticosteroid injection technique-With a 10cc syringe, $2 \mathrm{ml}$ of methylprednisolone acetate (Depo Medrol) is diluted with $5 \mathrm{ml}$ of plain lignocaine. Under aseptic precaution the lateral epicondyle is palpated and the injection is given perpendicular to it at the common extensor origin.

- PRP Technique: A sample of venous blood is collected from the patient's cubital vein and mixed with $4-5 \mathrm{ml}$ of anticoagulant (CPDA) to make a total volume of $20 \mathrm{ml}$. It is then equally divided into 4 vacuum containers and centrifuged at 3500rpm for7 minutes. The Buffy coat is aspirated from each container and the collected sample is spun at 3000rpm for 5 minutes. Once again the Buffy coat is collected in a $5 \mathrm{ml}$ syringe ${ }^{1}$.

- Patients are advised no strenuous activities of the affected for 7 days after the injection with only oral tramadol with paracetamol given for analgesia.

- Corticosteroid injection technique-With a 10cc syringe, $2 \mathrm{ml}$ of methylprednisolone acetate (Depo Medrol) is diluted with $5 \mathrm{ml}$ of plain lignocaine. Under aseptic precaution the lateral epicondyle is palpated and the injection is given perpendicular to it at the common extensor origin.

- PRP Technique: A sample of venous blood is collected from the patient's cubital vein and mixed with $4-5 \mathrm{ml}$ of anticoagulant (CPDA) to make a total volume of $20 \mathrm{ml}$. It is then equally divided into 4 vacuum 
containers and centrifuged at 3500rpm for 7 minutes. The Buffy coat is aspirated from each container and the collected sample is spun at 3000rpm for 5 minutes. Once again the Buffy coat is collected in a $5 \mathrm{ml}$ syringe ${ }^{1}$.
- Patients are advised no strenuous activities of the affected for 7 days after the injection with only oral tramadol with paracetamol given for analgesia.

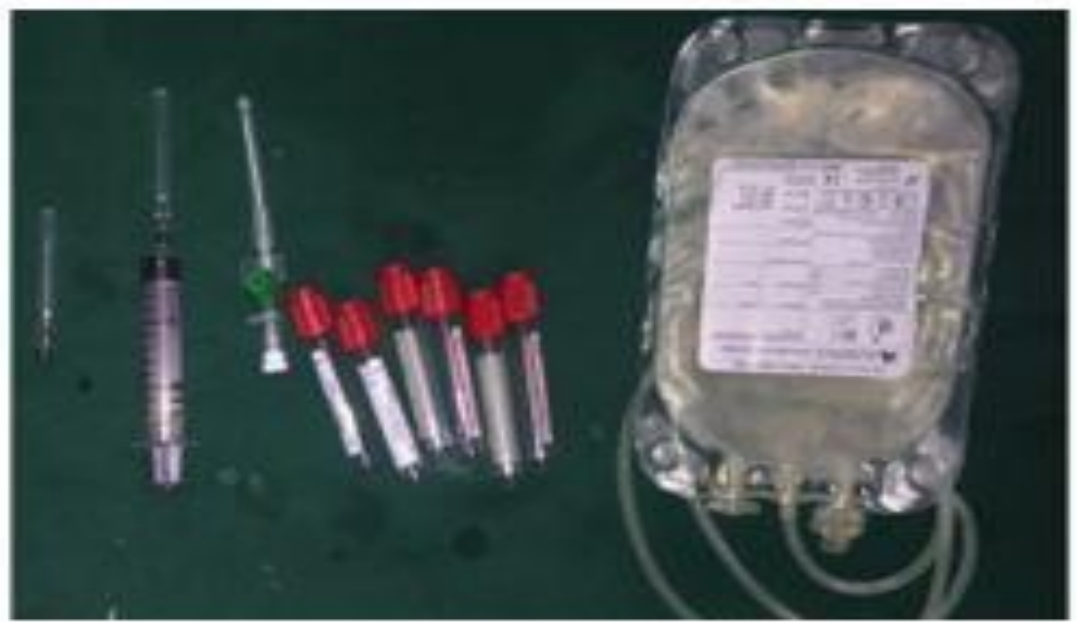

Figure 1: Blood bag, vacutainers, I.V. cannula used for PRP

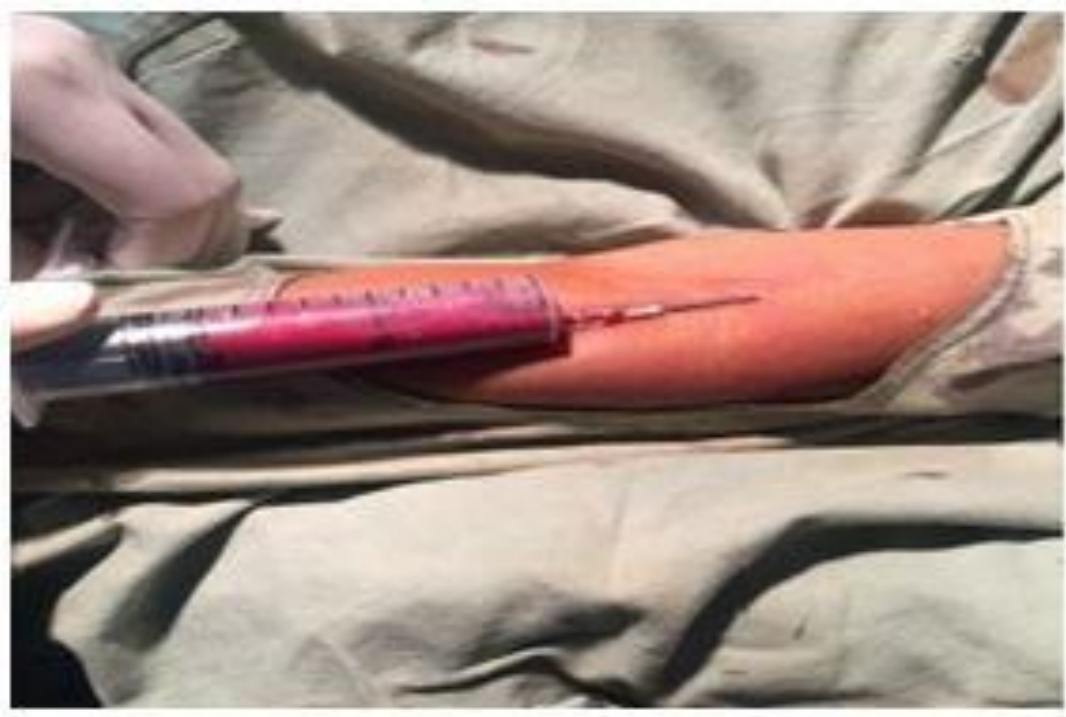

Figure 2 : Procedure of drawing blood

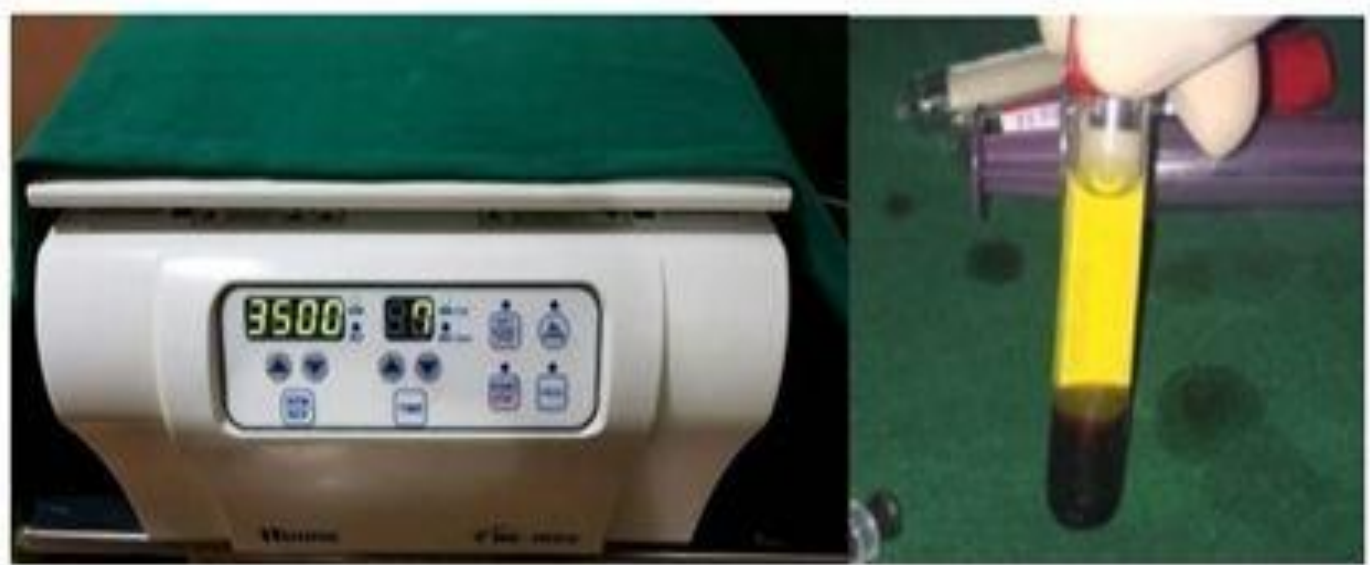

Figure 3: Centrifuge first spin: 3500RPM x7 minutes 


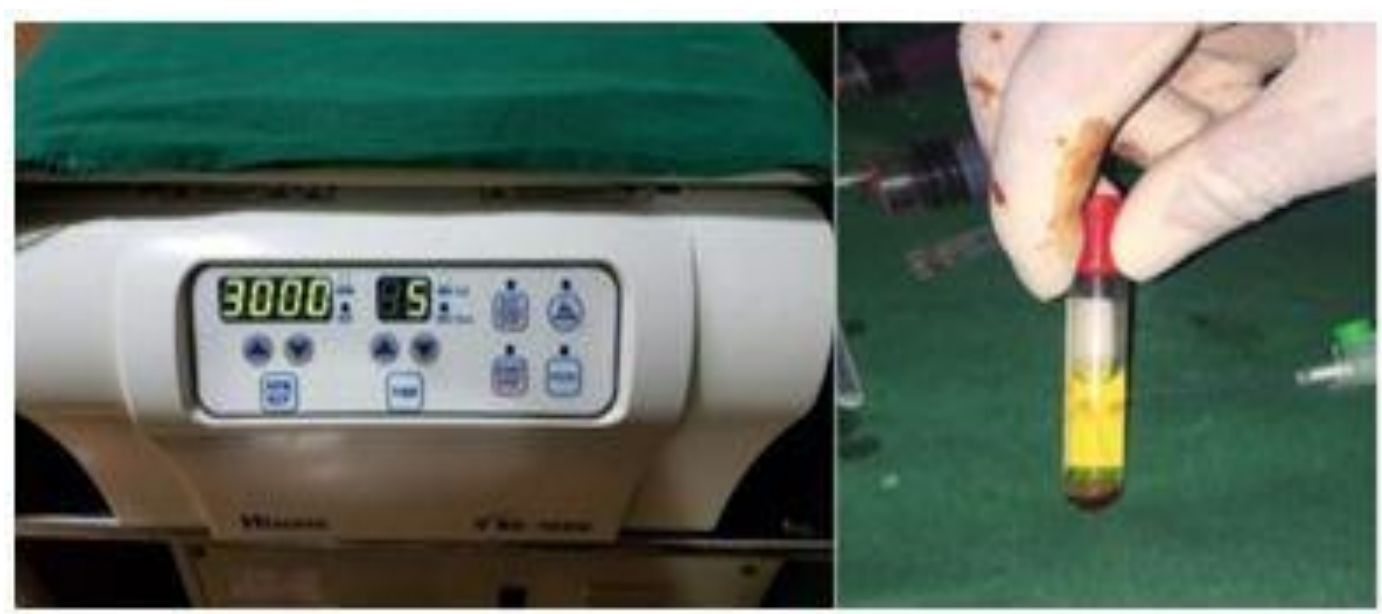

Figure 4: Centrifuge second spin: 3000RPM x5 minutes

Patients were then assessed by VAS (Visual Analogue Score) and Liverpool Elbow Scoring (LES) System at 3 weeks, 6 weeks and 6 months.

\section{Results}

VAS and LES scores at pre injection and at 6 weeks in PRP group showed a decrease at 6 weeks with a mean difference of 1.6 and increase in LES score score with mean difference 6.75 which is statistically significant where $\mathrm{p}$ value $<0.001$.

VAS and LES scores at pre injection and at 3 months in PRP group showed a decrease at 3 months with a mean difference of 3 and increase in LES score with mean difference of 8.4 which is statistically significant where $\mathrm{p}$ value $<0.001 \mathrm{VAS}$ and LES scores at pre injection and at 6 months in PRP group showed a decrease at 6 months with a mean difference of 5.95 and increase in LES score with a mean difference of 11.65 which is statistically significant where $\mathrm{p}$ value $<0.001$.

VAS and LES scores at pre injection and at 6 weeks showed a decrease at 6 weeks with a mean difference of 1.67 and an increase in LES with a mean difference of 2.45 which is statistically significant where $\mathrm{p}$ value $<0.001$.
VAS and LES scores at pre injection and at 3 months showed a decrease at 3 months with a mean difference of 2.05 and an increase in LES score with a mean difference of 2.675 which is statistically significant where $\mathrm{p}$ value $<0.001$

VAS and LES scores at pre injection and at 6 months showed a decrease at 6 months with a mean difference of 2.65 and an increase in LES score with a mean difference of 3.95 which is statistically significant where $\mathrm{p}$ value $<0.001$.

VAS and LES was compared between preinjection and at 6 weeks which was higher in corticosteroids with t value of 0.39 but LES was higher in PRP group with a $t$ value of 6.007 respectively and was statistically not significant with a p value 0.699 .

VAS and LES was compared between preinjection and at 3 months which was higher in PRP group with a $t$ value of 2.23 and $t$ value of 7.627 respectively and was statistically significant with a $\mathrm{p}$ value $<0.001$

VAS and LES was compared between preinjection and at 6 months which was higher in PRP group with a $t$ value of 7.403 and with a $t$ value of 9.503 respectively and was statistically significant with a p value $<0.001$ 

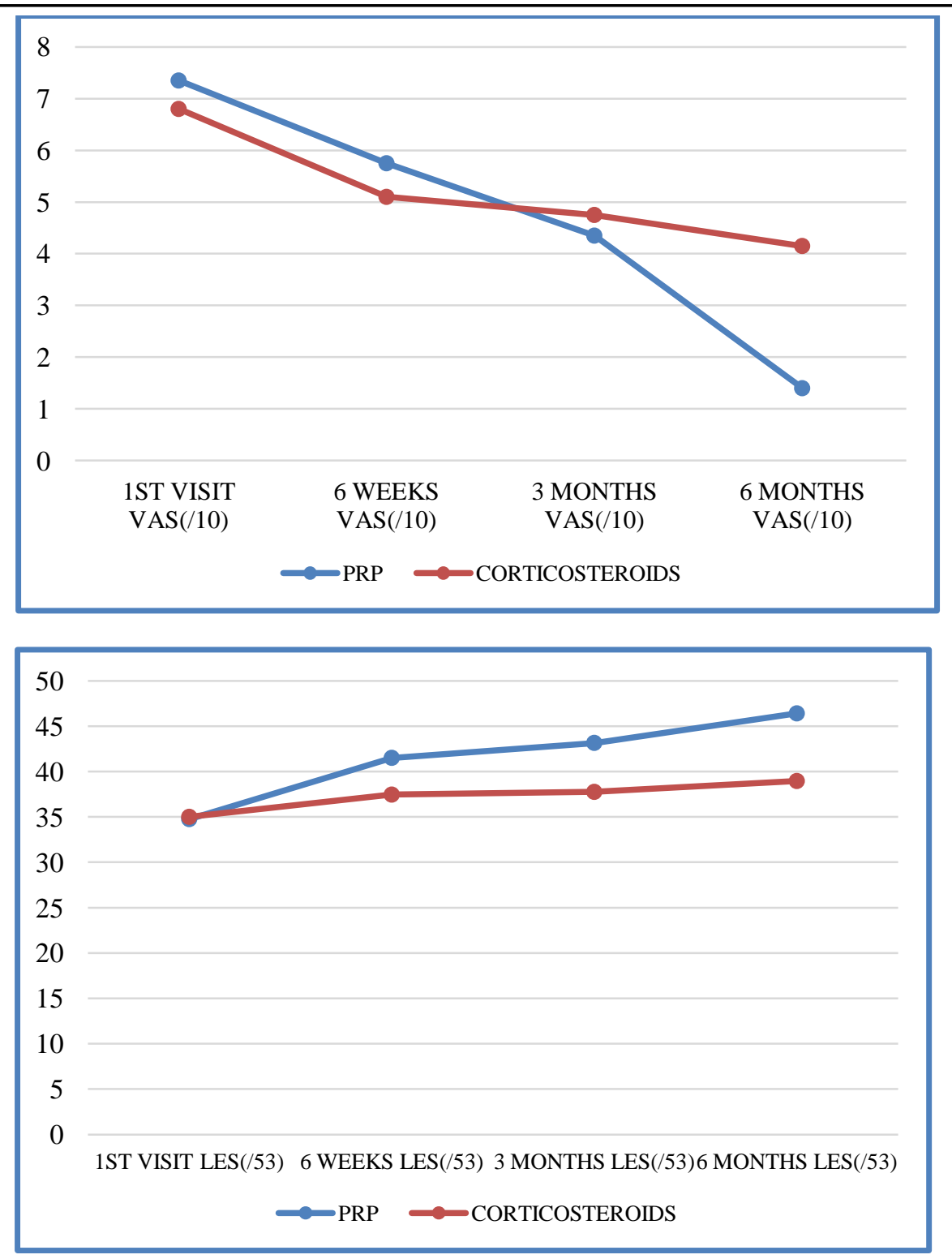

\section{Discussion}

Our study has assessed pain and functional outcome in patients who were given PRP or corticosteroids in the treatment of tennis elbow.

Of the 20 patients treated with PRP injections VAS show a gradual improvement in the follow up period of 6 weeks (5.75), 3 months (4.35), 6 months (1.4). The results were statistically significant with $\mathrm{P}$ value $<0.05$.

As seen in the above table our study shows similar results with Thasnas et al study, however the $\mathrm{p}$ value for improvement between the successive follow up is statistically significant across all the follow up in our study but only between preinjection and 6 weeks follow up in Thasnas et al study ${ }^{1}$.
LES also showed a gradual improvement in the follow up period of 6 weeks (41.5), 3 months (43.15), 6 months (46.4). The results were statistically significant with $\mathrm{P}$ value $<0.05$. As seen in the above table, our study shows similar results with Thasnas et al study, however the $\mathrm{p}$ value for improvement between the successive follow up is statistically significant across all the follow up in our study but not significant in Thasnas et al study ${ }^{1}$.

Our study also showed similar results with Peerbooms JC et al study, showing in improvement in VAS score in both PRP and corticosteroid group with corticosteroid group better initially and the declined while PRP group progressively improved ${ }^{4}$. 
An intergroup comparison was done to compare which injection is most effective for the treatment of lateral epicondylitis. The results of the intergroup comparison show that VAS score in corticosteroid group is better than PRP group within first 6 weeks. However VAS was better with PRP group in successive follow up and was statistically significant. The intergroup comparison results of LES score in PRP group was statistically significant than the corticosteroid group in all the follow up periods.

The improved results within the group between the follow ups which is statistically significant in our study compared to other studies is possibly because of the larger volume of PRP which we are injecting compared to the other study in which they give $3 \mathrm{ml}$ while we give $5 \mathrm{ml}$. While giving this $5 \mathrm{ml}$, we probably end up having more quantity of growth factors (Transforming Growth Factor, Vascular Endothelial Growth Factor, Fibroblast Growth Factor, Epidermal Growth Factor, Platelet Derived Growth Factor) ${ }^{7}$. This possibly is giving a better results compared to other studies. However in the same time, there would be possibly more pain because of large amount of PRP injecting to the patients on the day of injection. However our methodology did not consider this and we have not recorded the VAS score on the day of injection to know whether there was increase amount of pain in patients treated with $5 \mathrm{ml}$ of PRP injection

Our study showed that there was statistically significant better functional and vas score in patients treated with either PRP or corticosteroids, however, PRP group had more functional outcome and better pain relief compared to corticosteroids.

\section{Conclusion}

From our study it is evident that PRP provides symptomatic relief in the treatment of lateral epicondylitis, showing significant decrease in VAS score and increase in LES score.
Similarly corticosteroids provides symptomatic relief in the treatment of lateral epicondylitis, showing significant decrease in VAS score and increase in LES score.

When comparing PRP and corticosteroid injections, PRP proved to be more effective modality in the treatment of lateral epicondylitis with a statistically significant better functional outcome and better pain relief.

\section{References}

1. Thanasas C, Papadimitriou G, Charalambidis C, Paraskevopoulos I, Papanikolaou A. "Platelet-rich plasma Vs autologous whole blood for treatment of chronic lateral elbow epicondylitis: ARCT". Am J Sports Med, August 2011 39:2130- 2134.

2. Shiri R, Viikari-juntura E, Vareven $H$, HeliovaaraM."Prevalence and determinants of lateral and medial epicondylitis; a population study". Am J Epidemiology 2006,1;164 (11) : 1065-74.

3. Zeisig E, Fahlstrom M, Ohberg L, Alfredson H.“A 2 - year sonographic follow -up after intratendinous injection therapy in patients with lateral epicondylitis". Br J Sports Med, 2008 42(4):267-71.

4. Peerbooms JC, Sluimer J, Brujin DJ, Gosens T. "Positive effect of an autologous platelet concentrate in lateral epicondylitis in a doubleblind randomized controlled trial: platelet-rich plasma versus corticosteroid injection with a 1-year follow-up."Am J Sports Med 2010 38(2):255-62.

5. Sampson S, Gerhardt M, Mandelbaum B. Platelet rich plasma injection grafts for musculoskeletal injury. Cur rev of musculoskeletal med. 2008.1:165-174. 